\title{
Deterministic Sea Waves Prediction Using Mixed Space-Time Wave Radar Data
}

\author{
M. Al-Ani, J. Christmas, And M. R. Belmont \\ University of Exeter, Exeter, United Kingdom \\ J. M. DUNCAN AND J. DUNCAN \\ Defence Equipment and Support, Ministry of Defence, Bristol, United Kingdom \\ B. FERRIER \\ Dynamic Interface Laboratory, Hoffman Engineering Corporation, Stamford, Connecticut
}

(Manuscript received 25 August 2017, in final form 21 February 2019)

\begin{abstract}
A number of maritime operations can benefit from a short-term deterministic sea wave prediction (DSWP). Conventional X-band radars have recently been shown to provide a low-cost convenient source of twodimensional wave profile information for DSWP purposes. However, such rotating radars typically introduce temporal smearing into the data, which introduces errors when traditional Fourier transform-based wave prediction methods are used. The authors report on a new approach for DSWP that avoids such errors. Furthermore, it is not susceptible to the condition number problems that arise with any form of direct or indirect inversion-based approaches. Extensive numerical analyses are conducted to illustrate the effect of the mixed space-time nature of the data on DSWP and the efficiency of the proposed technique to handle it.
\end{abstract}

\section{Introduction}

It is of major international maritime interest to increase the sea state under which a wide range of launch and recovery operations can be safely undertaken (Pourzanjani et al. 1992; Ferrier et al. 2000; Crossland et al. 2009; Ferrier et al. 2013; Naaijen et al. 2016). Typical tasks include the launch and recovery of fixed and rotary wing aircraft; small, surface, or submersible vehicles, such as rigid-hulled inflatable boats (RHIBs); and cargo, fuel, and personnel transfer systems between vessels and from vessels onto offshore structures.

Deterministic sea wave prediction (DSWP) is a relatively new branch of marine science which unlike traditional statistical estimation of sea wave properties (Tucker and Pitt 2001) aims to predict the actual profile of the sea surface and its evolution, typically in the time scale of $60 \mathrm{~s}$ ahead. Based on two common elements, DSWP can play a vital role in extending the sea states under which critical maritime operations can be undertaken. First, while the overall execution of many maritime operations may take a significant amount of time,

\footnotetext{
Corresponding author: M. Al-Ani, m.t.a.al-ani@exeter.ac.uk
}

the key wave-height critical subtasks that actually limit the sea state under which they can be carried out are short, typically less than one minute. Second, a fundamental property of developed seas is that consecutive low waves group together leading to periods, known as quiescent periods, where the wave heights are below a certain threshold. DSWP has the capability of identifying the occurrence of the quiescent periods, allowing the wave-limited tasks to be safely carried out under sea conditions that based upon sea-state statistics alone would be considered unsafe. The deterministic prediction of such periods is termed quiescent period prediction (QPP), and it is the driver behind the work of Ferrier et al. (2000), Crossland et al. (2009), and Ferrier et al. (2013).

The main wave systems of DSWP interest arise from combinations of few remote storms whose spectra are downshifted to long wavelengths, and can significantly affect a vessel. Such wave systems have intermediateto narrowband spectra and exhibit the highest waves groupness (Longuet-Higgins 1984), resulting in long quiescent periods that are of particular interest. This is opposed to the locally produced wind waves, with broader spectra and hence less groupness. Additionally, for the 
locally produced wind waves, the local wind conditions in themselves could have a considerable impact on operations, limiting the benefits of DSWP/QPP in the first place in many cases.

In Belmont et al. (2014), we explored the feasibility of using data from an experimental wave-profiling radar in achieving DSWP. Two forms of DSWP were described: a fixed-point system based on a restricted set of wave directions, from which we obtained some success, and a fully two-dimensional technique that required further development. The main finding was that using wave-profiling radar for two-dimensional DSWP offers promise but requires new approaches to deterministic wave prediction that explicitly incorporate the mixed space-time nature of the data. One such method is developed here and is found to offer significant promise, not only because it accepts the mixed space-time data but also because it avoids the effects of the poorcondition number behavior associated with the type of matrices involved in DSWP (e.g., Connell et al. 2015; Alford et al. 2015).

The paper is organized as follows. In section 2, preliminaries about DSWP are presented. Section 3 includes a discussion on the adopted sea model (section $3 \mathrm{a}$ ), the description of the proposed technique of calculating the coefficients of the model from the mixed space-time data (section $3 \mathrm{~b}$ ), the selection of the prefixed parameters of the model (section 3c), and a discussion on the important topic of directional ambiguity in DSWP (section 3d). In section 4 , two sets of numerical analyses are detailed to illustrate the full potential of the proposed technique. By using idealized mixed space-time data, the first set focuses on the effect of the mixed space-time nature of the data on the traditional approaches and the ability of the proposed technique to handle it. The second set aims at testing the technique on simulated data with typical radar-degrading artifacts incorporated. Finally, conclusions are drawn in section 5 .

\section{Fundamentals of DSWP}

DSWP is the prediction of the actual shape of the sea surface (Morris et al. 1992, 1998; Belmont et al. 2003; Belmont 2010; Belmont et al. 2014) and its temporal evolution for a short time into the future, typically at the location of a moving vessel. Unlike statistical prediction of the sea surface (Tucker and Pitt 2001) which concerns time scales of up to days, DSWP is very short term, typically around $60 \mathrm{~s}$. The prediction time available is simply set by the time taken for waves to propagate from the location where they are measured to the prediction site. The relationship between the available prediction time, prediction space, and the properties of the measurement process can be described by the so-called space-time diagrams (Abusedra and Belmont 2011; Edgar et al. 2000; Naaijen et al. 2014; Qi et al. 2018) which yield all the necessary information. These diagrams show that there is always a user-defined compromise to be achieved between prediction time, prediction space, and accuracy.

DSWP can generally be achieved in two different ways. The first approach (adopted, e.g., in Belmont et al. 2007; Naaijen and Wijaya 2014; Hilmer and Thornhill 2015) is to (i) calculate the spectral coefficients of the measured wave data (mainly using classical Fourier transform techniques), (ii) phase shift the obtained spectral coefficients by the prediction time and the angular frequency defined by the dispersion relationship, and (iii) spectral inversion of the shifted spectral coefficients to construct the predicted sea surface elevation. (When predicting wave-induced vessel motions, the frequency domain vessel models can use the spectral coefficients directly before inversion; e.g., Connell et al. 2015.) The second approach is to directly convolve the measured wave data region with an appropriate form of the impulse response of the sea. This approach reduces the computational complexity by producing the wave prediction in one step, as opposed to the three steps involved in the first approach. However, it needs care because of certain pathological properties of the impulse response of the sea (Belmont et al. 2006), and hence the first approach is adopted here.

Several wave-sensing technologies that can provide the required resolution and coverage have been explored in DSWP: wave sensor buoys, scanning waveprofiling lidar, and ship-mounted wave radar. Wave buoys are viable around fixed installations but are not realistic for freely operating vessels. They do also have a research role. Furthermore, wave buoys return single-point measures of the wave system, which are not sufficient to describe highly two-dimensional seas. Scanning wave-profiling lidar systems (Belmont et al. 2007) have the necessary functionality, but are as yet at the research stage, and the only marinized versions commercially available have too short a range. This leaves wave-profiling radars as the most attractive sensing technology for DSWP. Conventional vessel-mounted wave radar returns statistical wave data (sea surface roughness). However, the OceanWaveS Wave and Surface Current Monitoring System (WaMoS) II waveprofiling radar (Reichert et al. 1999) offers the prospect of measuring the actual sea surface profile over an area of a few square kilometers around a vessel, based on the standard X-band radar carried by ships for safe navigation purposes. In Hilmer and Thornhill (2014), the accuracy of WaMoS II at rendering sea surface 
elevation was evaluated against a wave buoy, where the majority of correlation values exceeded 0.87 .

Typically wave radar data are captured using a mechanically rotated antenna, and hence the time stamps associated with the data points in a single radar scan vary by up to the rotation period. Thus, the data have an inherently mixed space-time character. The easiest solution is to simply ignore this temporal smearing and treat the radar scan data as a spatial "snapshot." This constitutes a considerable source of error in the prediction, as will be seen in section 4 . In the next section, we present the technique that can accept the mixed space-time data.

\section{Mixed space-time DSWP}

\section{a. The adopted sea model}

The useful range of wave-profiling radars is at most $3 \mathrm{~km}$ which restricts the maximum wave propagation distances and hence the predict-ahead times. Clearly, all measurements and computations associated with DSWP directly subtract from the wave propagation time and hence the predict-ahead time. Hence, these tasks must all be completed in relatively short times. Thus, while there has been some interest in deterministic prediction of the propagation of nonlinear waves (e.g., Prislin et al. 1997; Blondel et al. 2008), the associated computational costs are far too large to allow nonlinear wave-based DSWP for all but highly one-dimensional seas. Consequently, the wave propagation models used in DSWP here are restricted to linear wave theory. However, this is not a strong limitation as the linear wave models can sufficiently describe the waves of most interest in DSWP (i.e., waves of remote storms whose spectra are downshifted to long wavelengths, as discussed in the introduction), especially with the time scales of multiple tens of seconds involved in DSWP.

The standard linear wave model of the sea surface elevation $h(x, y, t)$ at the spatial coordinate $(x, y)$, and temporal coordinate $t$, is given by

$$
\begin{aligned}
h(x, y, t)= & \sum_{r=1}^{R} \sum_{n=1}^{N} c_{n, r} \cos \left[k_{n} x \cos \left(\theta_{r}\right)+k_{n} y \sin \left(\theta_{r}\right)\right. \\
& \left.-\omega_{n} t+\varphi_{n, r}\right],
\end{aligned}
$$

where $N$ is the number of spectral components in the propagation direction $\theta_{r}, R$ is the number of directions, and $c_{n, r}$ is the magnitude spectral coefficient at the wavenumber $k_{n}$ and angular frequency $\omega_{n}$ of initial phase $\varphi_{n, r}$ in the direction $\theta_{r}$. The proposed technique of calculating the magnitudes and phases of the spectral coefficients $\left(\left\{c_{n, r}\right\}\right.$ and $\left\{\varphi_{n, r}\right\}$, respectively) from the mixed space-time data is discussed in the following subsection. Selecting the preset parameters, $\left\{k_{n}\right\}_{n=1}^{N}$ and
$\left\{\theta_{r}\right\}_{r=1}^{R}$ in (1) are discussed in section 3c. The angular frequency $\omega_{n}$ is determined from the wavenumber $k_{n}$ via the deep-water dispersion relationship.

\section{b. Model coefficients calculation}

The mixed space-time data problem that we tackle here is related to the existing body of literature of spectral analysis of unevenly spaced data. The intuitive solution for the above two problems is the least squares fitting of the data to all the spectral components simultaneously. This would require inverting a poorly conditioned matrix (or equivalently solving a Vandermonde system) which is computationally prohibitive with the inevitable errors involved in the data and modeling being highly magnified by virtue of the very large condition numbers. This is especially so given the large size of the two-dimensional spatial wave profile data involved. Alternative approaches could be based on interpolation or approximation. However, the performance of these approaches will be determined by the properties of the data and the choice of the interpolation/approximation function. Our aim here is to develop a general approach that is capable of handling the mixed space-time data at sensible computational complexity. The technique described will be based on the single-point least squares estimation that was originally developed by Vaníček (1969) and formed the basis for other methods in the theory of least squares spectral analysis such as the well-known Lomb-Scargle periodogram (Lomb 1976; Scargle 1982).

The problem of inverting a poorly conditioned large matrix is avoided by simply examining one wavenumber at a time independently of the others. This amounts to defining the spectrum as the independent contribution of each wave component, and it is equivalent to ignoring the linear dependence between different wave components in $h(x, y, t)$. This is sensible given the theory of Gaussian processes of sea waves (Tucker et al. 1984) which states that $c_{n, r}$ are independent random variables chosen from Rayleigh distribution and $\varphi_{n, r}$ are also independent with uniform distribution on $[0,2 \pi)$.

Writing

$$
\begin{aligned}
& a_{n, r} \triangleq c_{n, r} \cos \left(\varphi_{n, r}\right), \\
& b_{n, r} \triangleq-c_{n, r} \sin \left(\varphi_{n, r}\right),
\end{aligned}
$$

(1) can be written as

$$
\begin{aligned}
h(x, y, t)= & \sum_{r=1}^{R} \sum_{n=1}^{N}\left\{a_{n, r} \cos \left[k_{n} x \cos \left(\theta_{r}\right)+k_{n} y \sin \left(\theta_{r}\right)-\omega_{n} t\right]\right. \\
& \left.+b_{n, r} \sin \left[k_{n} x \cos \left(\theta_{r}\right)+k_{n} y \sin \left(\theta_{r}\right)-\omega_{n} t\right]\right\} .
\end{aligned}
$$


Subsequently (4) will be rewritten using the more compact nomenclature:

$$
\begin{aligned}
& \alpha_{n, r}(x, y, t) \triangleq \cos \left[k_{n} x \cos \left(\theta_{r}\right)+k_{n} y \sin \left(\theta_{r}\right)-\omega_{n} t\right], \\
& \beta_{n, r}(x, y, t) \triangleq \sin \left[k_{n} x \cos \left(\theta_{r}\right)+k_{n} y \sin \left(\theta_{r}\right)-\omega_{n} t\right] .
\end{aligned}
$$

Furthermore, we write, $z=(x, y) \in \mathbb{R}^{2}$. The sea elevation discrete measurements from one radar scan is then denoted by $\left\{h_{s}\left(z_{m}, t_{s, m}\right)\right\}_{m=1}^{M}, z_{m}=\left(x_{m}, y_{m}\right)$, where $M$ is the size of the data of the $s$ th scan. For a single scan, the sequence $\left\{t_{s, m}\right\}_{m=1}^{M}$ range over the interval, $\left(\tau_{s}, \tau_{s}+\Delta t\right]$, where $\tau_{s}$ is the start time of the sth scan and $\Delta t$ is the rotation period of the radar antenna. The set of spatial sample locations is $\left\{\left(x_{m}, y_{m}\right)\right\}_{m=1}^{M}$.

The coefficients $a_{n, r}$ and $b_{n, r}$ can be determined by solving the following minimization tasks

$$
\begin{aligned}
& \min _{a_{n, r}, b_{n, r}} \sum_{m=1}^{M}\left[a_{n, r} \alpha_{n, r}\left(z_{m}, t_{s, m}\right)+b_{n, r} \beta_{n, r}\left(z_{m}, t_{s, m}\right)\right. \\
& \left.\quad-h_{s}\left(z_{m}, t_{s, m}\right)\right]^{2} .
\end{aligned}
$$

The solution is given by

$$
\left[\begin{array}{l}
\hat{a}_{n, r} \\
\hat{b}_{n, r}
\end{array}\right]=\mathbf{H}^{-1} \mathbf{f},
$$

where

$$
\begin{aligned}
\mathbf{H} & =\sum_{m=1}^{M}\left[\begin{array}{l}
\alpha_{n, r}\left(z_{m}, t_{s, m}\right) \\
\beta_{n, r}\left(z_{m}, t_{s, m}\right)
\end{array}\right]\left[\alpha_{n, r}\left(z_{m}, t_{s, m}\right) \beta_{n, r}\left(z_{m}, t_{s, m}\right)\right] \\
\mathbf{f} & =\sum_{m=1}^{M}\left[\begin{array}{l}
\alpha_{n, r}\left(z_{m}, t_{s, m}\right) \\
\beta_{n, r}\left(z_{m}, t_{s, m}\right)
\end{array}\right] h_{s}\left(z_{m}, t_{s, m}\right)
\end{aligned}
$$

where the magnitudes and phases of the components are recovered via (2) and (3).

The reasoning behind this approach, based on Scargle (1982), is that the spectral components within the data associated with a particular wave component of wavenumber and direction $\left(k_{n}, \theta_{r}\right)$ will be in phase with the components in (10) of the same wavenumber and direction and make a large contribution to the sums. But at different $\left(k_{n}, \theta_{r}\right)$, such components produce randomly positive and negative terms in the sums of (10) that tend to cancel. In other words, while the pairs of vectors evaluated from (5) and (6) may not be entirely linearly independent, the degree of correlation among them is sufficiently weak to justify ignoring mutual correlation effects (at least when they are not too closely spaced). It is significant to note that if the variation in time is ignored and $t_{s, m}$ is assumed to be a fixed value in the trigonometric components (5) and (6), their values in (9) would actually be orthogonal to each other and (7) would reduce to the classical inner product of discretetime Fourier transform. Thus, the conventional discretetime Fourier transform can be viewed as a special case of this more general mixed space-time technique.

We note that the fitting of (8) is for each pair of the wave components, which only requires inverting $2 \times 2$ matrices. The arithmetic complexity of computing a spectral coefficient according to the present approach is $O(M)$. This is asymptotically equivalent to the standard discrete-time Fourier transform that would be used where an FFT cannot be deployed.

\section{c. Model preset parameters}

The natural spatial locations of the radar measurements are distributed in a polar form. [However, the model (9) puts no assumption on the distribution of the measurements, and the data from a Cartesian grid could also be used.] The wavenumbers $\left\{k_{n}\right\}_{n=1}^{N}$ in (1) are set to uniformly span over the longest and shortest waves to represent. The angular directions $\left\{\theta_{r}\right\}_{r=1}^{R}$ are evenly distributed over a half plane (a discussion on the motive is in section 3d). Using one whole radar scan of data, the wavenumber resolution in (1) is defined by $\pi / \gamma$, where $\gamma$ is the radius of the radar coverage. The directional resolution depends on the choice of the maximum wavenumber $k_{N}$ and is defined by $2 \pi / \gamma k_{N}$. The maximum wavenumber $k_{N}$ can be set as high as $\pi / \mu$, where $\mu$ is the distance between adjacent samples along the radar radial spoke, and the minimum wavenumber $k_{1}$ can be set as low as $\pi / \gamma$.

The radar measurements usually span a large coverage with relatively high resolution, and hence a wide spectral sea model with large $R$ and $N$ can be built. However, in many cases, the wave spectrum and the frequency response of the wave-driven vessel are limited to a narrower range of wavenumbers. Hence, for reasons of computational efficiency, it is important to restrict the calculations to only those wavenumbers $\left\{k_{n}\right\}_{n=1}^{N}$ that are relevant to the context at hand. Such restriction in $\left\{k_{n}\right\}_{n=1}^{N}$ (i.e., in $N$ and $R$, as the latter depends on $k_{N}$ ) with minimal compromise in the quality of prediction can be confirmed by, for example, performing a simple self-consistency check on the prevailing case: 1) identify/ calculate the restricted spectral coefficients, 2) perform an inversion with no phase shifting, and 3) compare the outcome with the original measurements.

It is important to note that the summations involved in (1) and in the least squares estimation process in (10) involve quantities that are functions of polar coordinates. 
Hence, contribution to each term in the two-dimensional summations must be scaled by the corresponding discrete approximation to the differential area element. Given that the angular step size is constant, this means that each term is weighted by the radial coordinate.

\section{d. Directional ambiguity}

A wave propagation model, whether its coefficients are estimated from one true snapshot of the sea surface profile or from mixed space-time data obtained from one radar rotation, can suffer from $\pi$ radian directional ambiguity. From a purely algebraic perspective, the directional ambiguity can be resolved by using two spectral estimates derived from two radar scans [with some limitations as discussed in Atanassov et al. (1985)]. However, for the large developed seas, which are of most interest in practical maritime applications of DSWP, the wave systems are produced by a very limited number of remote storms. Consequently, it is very unlikely that two significant wave systems will be contributing whose directions differ by close to $\pi$ radians and hence there is no actual directional ambiguity in this case. Thus, a key part of DSWP is a preliminary inspection of the dominant wave directions using directional power spectral density obtained using standard techniques. Such directional power spectra would be determined as a background computational task in a DSWP system which is updated every few minutes.

In cases where the directional ambiguity is relevant, the obvious solution is the fully three-dimensional Fourier transform, based on a number of radar scans (Young et al. 1985), or equivalently applying the minimization of (7) to data from a number of scans. While this approach is well suited for determining directional power spectra for statistical applications, the same cannot be said for producing the wave profiles required for DSWP. In DSWP, using multiple scans (especially when the number of scans is large) introduces undesired windowing effect on parts of the predicted wave profiles. This effect is not pronounced in statistical applications where we are only concerned with the spectral energy in the spectral bins (i.e., no inversion to sea surface elevation). Analysis of this windowing effect on DSWP will be dealt with in a future publication. In this work, we focus on determining the prediction using one radar scan where the wave storms are known to originate from one half plane.

\section{Numerical evaluation}

Two sets of experiments are considered here to comprehensively evaluate the proposed technique. In the first set, idealized mixed space-time wave data are simulated based upon a radar scanner of rotation period of a few seconds. We compare the prediction quality of the presented technique, where the actual time stamp attached to each spatial location is used, with the prediction quality of the traditional Fourier method where a single time stamp is associated/approximated with each scan. The aim here is to focus on the prediction error in ignoring the continuous time stamp in the traditional method, and the capability of the proposed technique to handle it. Thus, the used data are idealized with no measurement noise, spatial sampling jitter, directional attenuation, interpolation error (in case of the traditional Fourier method), etc.

In the second case, we conduct a more realistic experiment, where typical radar-measuring artifacts are imposed on the mixed space-time data. The aim is to demonstrate the capacity of the proposed method in a more practical setup. These artifacts include spatial sampling jitter, measurements noise, a dead zone at the vicinity of the radar, and the unreliable measurement of the waves that are not traveling toward or away from the radar.

To simulate the data in the experiments in both cases, we use the standard linear method of superposing sinusoidal components. The phases are generated from a uniform random distribution over $[0,2 \pi$ ) and in order to obtain waves with the correct runs statistics (as opposed to merely "sealike behavior"), the amplitudes of the components are obtained by sampling from a frequencydependent Rayleigh distribution (Tucker et al. 1984). The RMS value of the Rayleigh distribution at each frequency is obtained from the (square root of the) power spectral density. The magnitudes are weighed by the directional spreading curve. Before describing the data and the results of the experiments, we define the performance metrics that are used in the evaluation of the results here in the following subsection.

\section{a. Performance metrics}

The radar scans are denoted by $h_{s}=\left\{h_{s}\left(z_{m}, t_{s, m}\right)\right\}_{m=1}^{M}$; $s=1, \ldots, S$, where $S$ is total number of scans per experiment. Each scan is used respectively to generate predictions of the sea surface for period $\left(\tau_{s}, \tau_{s}+T\right]$, where $T$ is set to $120 \mathrm{~s}$. The prediction of the sea surface based on $h_{s}$ at prediction time instant $t_{s+p, m} \in\left(\tau_{s}, \tau_{s}+T\right], p=1, \ldots, P$, where $P=T / \Delta t$, is denoted by $\hat{h}_{s}\left(z_{m}, t_{s+p, m}\right)$. To assess the prediction accuracy, we evaluate the normalized correlation between the predicted wave profile for $\left(\tau_{s}, \tau_{s}+T\right]$ at spatial location $z_{m}$ and the "future" data within the same period and location. Assuming that the predicted and true wave profiles, $\left\{\hat{h}_{s}\left(z_{m}, t_{s+p, m}\right)\right\}_{p=1}^{P}$ and $\left\{h_{s+p}\left(z_{m}, t_{s+p, m}\right)\right\}_{p=1}^{P}$ 
TABLE 1. The performance of the traditional Fourier technique with a single time stamp for simulation testing I.

\begin{tabular}{|c|c|c|c|c|}
\hline Sea state & $\frac{\Delta t=1.5 \mathrm{~s}}{A_{\mathcal{T}}\left(z_{m}\right), B_{\mathcal{T}}\left(z_{m}\right), C_{\mathcal{T}}\left(z_{m}\right)}$ & $\frac{\Delta t=2 \mathrm{~s}}{A_{\mathcal{T}}\left(z_{m}\right), B_{\mathcal{T}}\left(z_{m}\right), C_{\mathcal{T}}\left(z_{m}\right)}$ & $\frac{\Delta t=2.5 \mathrm{~s}}{A_{\mathcal{T}}\left(z_{m}\right), B_{\mathcal{T}}\left(z_{m}\right), C_{\mathcal{T}}\left(z_{m}\right)}$ & $\frac{\Delta t=3 \mathrm{~s}}{A_{\mathcal{T}}\left(z_{m}\right), B_{\mathcal{T}}\left(z_{m}\right), C_{\mathcal{T}}\left(z_{m}\right)}$ \\
\hline $\begin{array}{l}\mathcal{H}=4.4 \mathrm{~m} \\
\mathcal{P}=5.9 \mathrm{~s}\end{array}$ & $0.93,0.54,0.45$ & $0.86,0.61,0.53$ & $0.72,0.88,0.79$ & $0.67,0.98,0.85$ \\
\hline $\begin{array}{l}\mathcal{H}=4.4 \mathrm{~m} \\
\mathcal{P}=7 \mathrm{~s}\end{array}$ & $0.94,0.47,0.41$ & $0.90,0.54,0.46$ & $0.82,0.69,0.61$ & $0.73,0.91,0.79$ \\
\hline $\begin{array}{l}\mathcal{H}=4.4 \mathrm{~m} \\
\mathcal{P}=7.9 \mathrm{~s}\end{array}$ & $0.95,0.41,0.33$ & $0.90,0.48,0.41$ & $0.86,0.52,0.48$ & $0.82,0.68,0.58$ \\
\hline $\begin{array}{l}\mathcal{H}=4.7 \mathrm{~m} \\
\mathcal{P}=8.7 \mathrm{~s}\end{array}$ & $0.96,0.39,0.30$ & $0.91,0.47,0.40$ & $0.88,0.51,0.44$ & $0.83,0.65,0.56$ \\
\hline $\begin{array}{l}\mathcal{H}=6 \mathrm{~m} \\
\mathcal{P}=9.4 \mathrm{~s}\end{array}$ & $0.96,0.43,0.28$ & $0.92,0.55,0.38$ & $0.90,0.63,0.43$ & $0.86,0.76,0.52$ \\
\hline $\begin{array}{l}\mathcal{H}=7 \mathrm{~m} \\
\mathcal{P}=9.9 \mathrm{~s}\end{array}$ & $0.97,0.48,0.27$ & $0.95,0.61,0.35$ & $0.91,0.70,0.41$ & $0.88,0.78,0.49$ \\
\hline
\end{tabular}

respectively, are zero centered, their correlation coefficients at location $z_{m}$ based on $h_{s}$ are given by

$$
\begin{aligned}
Q_{s}\left(z_{m}\right) & =\frac{\frac{1}{P} \sum_{p=1}^{P} \hat{h}_{s}\left(z_{m}, t_{s+p, m}\right) h_{s+p}\left(z_{m}, t_{s+p, m}\right)}{\hat{\sigma}_{s}\left(z_{m}\right) \sigma_{s}\left(z_{m}\right)}, \\
s & =1, \ldots, S-P
\end{aligned}
$$

where

$$
\begin{aligned}
& \hat{\sigma}_{s}\left(z_{m}\right)=\left[\frac{1}{P} \sum_{p=1}^{P}\left|\hat{h}_{s}\left(z_{m}, t_{s+p, m}\right)\right|^{2}\right]^{1 / 2}, \\
& \sigma_{s}\left(z_{m}\right)=\left[\frac{1}{P} \sum_{p=1}^{P}\left|h_{s+p}\left(z_{m}, t_{s+p, m}\right)\right|^{2}\right]^{1 / 2} .
\end{aligned}
$$

To produce an overall illustration of the results of (11), we average the coefficients at location $z_{m}$ across the scans; that is, $A\left(z_{m}\right)=1 /(S-P) \sum_{s=1}^{S-P} Q_{s}\left(z_{m}\right)$.

While the correlation coefficient demonstrates the accuracy of predicting the shape of the wave profile, it does not depict the accuracy in the amplitude of the predicted wave. To this end, the RMS errors at $z_{m}$,

$$
\begin{aligned}
\varepsilon_{s}\left(z_{m}\right) & =\left[\frac{1}{P} \sum_{p=1}^{P}\left|\hat{h}_{s}\left(z_{m}, t_{s+p, m}\right)-h_{s+p}\left(z_{m}, t_{s+p, m}\right)\right|^{2}\right]^{1 / 2}, \\
s & =1, \ldots, S-P
\end{aligned}
$$

must also be included in the evaluation, along with the standard deviations of the predicted wave profile (12) and true profile (13), to provide complementary information quantifying the correspondence of the two profiles (Taylor 2001). For an overall illustration of the above, we define the mean RMS error at location $z_{m}$ across the scans by $B\left(z_{m}\right)=1 /(S-P) \sum_{s=1}^{S-P} \varepsilon_{s}\left(z_{m}\right)$, and the mean normalized RMS error by $C\left(z_{m}\right)=$ $1 /(S-P) \sum_{s=1}^{S-P} \varepsilon_{s}\left(z_{m}\right) / \sigma_{s}\left(z_{m}\right)$.

\section{b. Simulation testing I}

The comparison is conducted over simulation experiments of various sea states and radar rotation periods. It is important to examine the effect of the radar rotation period $\Delta t$ as this determines the extent to which the "snapshot" approximation (using a single time stamp per scan) is valid. The various sea-state data are generated using (shifted) JONSWAP spectra with peakedness parameter of 3.3 and a spread function of 12 in the original Cartwright (1963) form leading to directional spectra spread under one half plane. As mentioned earlier, no sources of error such as measurement noise or radar measurement artifacts are introduced into the simulated data.

The simulated radar data are in polar form and cover a 2-km-radius circle. The spatial resolution in radial distance and angular direction are $2 \mathrm{~m}$ and $0.0063 \mathrm{rad}$, respectively. Each spoke of radar data at a particular direction has its unique time stamp. In the case of the proposed method, the actual time stamps associated with the spokes in the data are employed. The minimum and maximum wavenumbers are set to 0.0016 and $0.16 \mathrm{rad} \mathrm{m}^{-1}$, respectively. The resolutions in wavenumber and angular direction are set to $0.0016 \mathrm{rad} \mathrm{m}^{-1}$ and $0.02 \mathrm{rad}$, respectively.

In the case of the traditional methods, the polar data from the sth scan are first "perfectly interpolated" into a dense Cartesian grid of size $4 \mathrm{~km} \times 4 \mathrm{~km}$ and resolution $1 \mathrm{~m} \times 1 \mathrm{~m}$. Most importantly, a single time stamp is associated to the whole grid (i.e., $\tau_{s}+\Delta t / 2$ ) in order for the classical 2D FFT to be used. The areas of the grid that lie outside the circular coverage of the radar are set to zero. 
TABLE 2. The performance of the proposed technique with the actual time stamp for simulation testing I.

\begin{tabular}{|c|c|c|c|c|}
\hline Sea state & $\frac{\Delta t=1.5 \mathrm{~s}}{A_{\mathcal{T}}\left(z_{m}\right), B_{\mathcal{T}}\left(z_{m}\right), C_{\mathcal{T}}\left(z_{m}\right)}$ & $\frac{\Delta t=2 \mathrm{~s}}{A_{\mathcal{T}}\left(z_{m}\right), B_{\mathcal{T}}\left(z_{m}\right), C_{\mathcal{T}}\left(z_{m}\right)}$ & $\frac{\Delta t=2.5 \mathrm{~s}}{A_{\mathcal{T}}\left(z_{m}\right), B_{\mathcal{T}}\left(z_{m}\right), C_{\mathcal{T}}\left(z_{m}\right)}$ & $\frac{\Delta t=3 \mathrm{~s}}{A_{\mathcal{T}}\left(z_{m}\right), B_{\mathcal{T}}\left(z_{m}\right), C_{\mathcal{T}}\left(z_{m}\right)}$ \\
\hline $\begin{array}{l}\mathcal{H}=4.4 \mathrm{~m} \\
\mathcal{P}=5.9 \mathrm{~s}\end{array}$ & $0.99,0.1,0.07$ & $0.99,0.09,0.08$ & $0.99,0.1,0.07$ & $0.99,0.1,0.07$ \\
\hline $\begin{array}{l}\mathcal{H}=4.4 \mathrm{~m} \\
\mathcal{P}=7 \mathrm{~s}\end{array}$ & $0.99,0.08,0.06$ & $0.99,0.1,0.09$ & $0.99,0.06,0.05$ & $0.99,0.08,0.06$ \\
\hline $\begin{array}{l}\mathcal{H}=4.4 \mathrm{~m} \\
\mathcal{P}=7.9 \mathrm{~s}\end{array}$ & $0.99,0.1,0.06$ & $0.99,0.08,0.07$ & $0.99,0.1,0.08$ & $0.99,0.08,0.07$ \\
\hline $\begin{array}{l}\mathcal{H}=4.7 \mathrm{~m} \\
\mathcal{P}=8.7 \mathrm{~s}\end{array}$ & $0.99,0.13,0.1$ & $0.99,0.12,0.09$ & $0.99,0.11,0.08$ & $0.99,0.12,0.1$ \\
\hline $\begin{array}{l}\mathcal{H}=6 \mathrm{~m} \\
\mathcal{P}=9.4 \mathrm{~s}\end{array}$ & $0.99,0.15,0.11$ & $0.99,0.13,0.09$ & $0.99,0.12,0.08$ & $0.99,0.13,0.08$ \\
\hline $\begin{array}{l}\mathcal{H}=7 \mathrm{~m} \\
\mathcal{P}=9.9 \mathrm{~s}\end{array}$ & $0.99,0.17,0.08$ & $0.99,0.17,0.09$ & $0.99,0.16,0.08$ & $0.99,0.15,0.10$ \\
\hline
\end{tabular}

Each experiment with specific sea state and radar rotation period is run for $20 \mathrm{~min}$. A prediction of the sea elevation, for total prediction period of $120 \mathrm{~s}$, at the ship location is constructed every $30 \mathrm{~s}$ using the classical and the proposed methods. The quality of predictions from both approaches are shown in Tables 1 and 2 in terms of the mean correlation $A_{\mathcal{T}}\left(z_{m}\right)$, the mean RMS error $B_{\mathcal{T}}\left(z_{m}\right)$, and the mean normalized RMS error $C_{\mathcal{T}}\left(z_{m}\right)$. The radar rotation periods are varied between 1.5 and $3 \mathrm{~s}$ as shown in the first rows of the tables. The various sea states of the generated data are described in terms of the wave spectral parameters: the significant wave height $\mathcal{H}$ and mean period $\mathcal{P}$ in the first columns of the tables.

The results of the experiments are shown in Tables 1 and 2 and can be summarized as follows:

(i) Under all conditions the proposed method using fully time-resolved data performs significantly better than the traditional two-dimensional Fourier approach using a single time stamp per scan. (ii) As would be expected the single time stamp approach worsens as the radar rotation period increases (i.e., the snapshot approximation worsens) while there is no noticeable effect on the proposed method.

(iii) There is a very modest improvement in the single time stamp method with increasing the wave period which is to be expected and has been anecdotally noted previously.

\section{c. Simulation testing II}

In this subsection, we synthetically generate and test the prediction of wave data of a more practical setup. Artifacts are introduced in the degraded mixed spacetime data case by first spatially windowing the data in order to (i) simulate the dead zone of 200-m radius immediately surrounding the vessel and (ii) simulate the attenuation in the measured waves that are not traveling toward or away from the radar. In addition, random Gaussian-sampling jitter of standard deviation of $0.5 \mathrm{~m}$

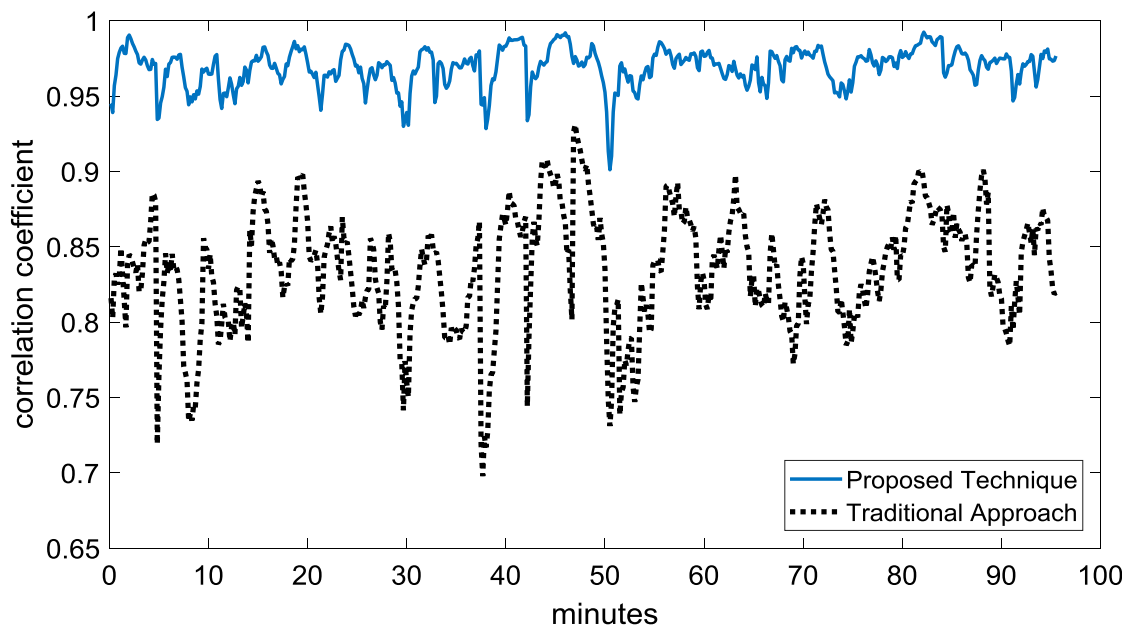

FIG. 1. The correlation coefficients at the ship location for simulation testing II. 
a) The Traditional Approach

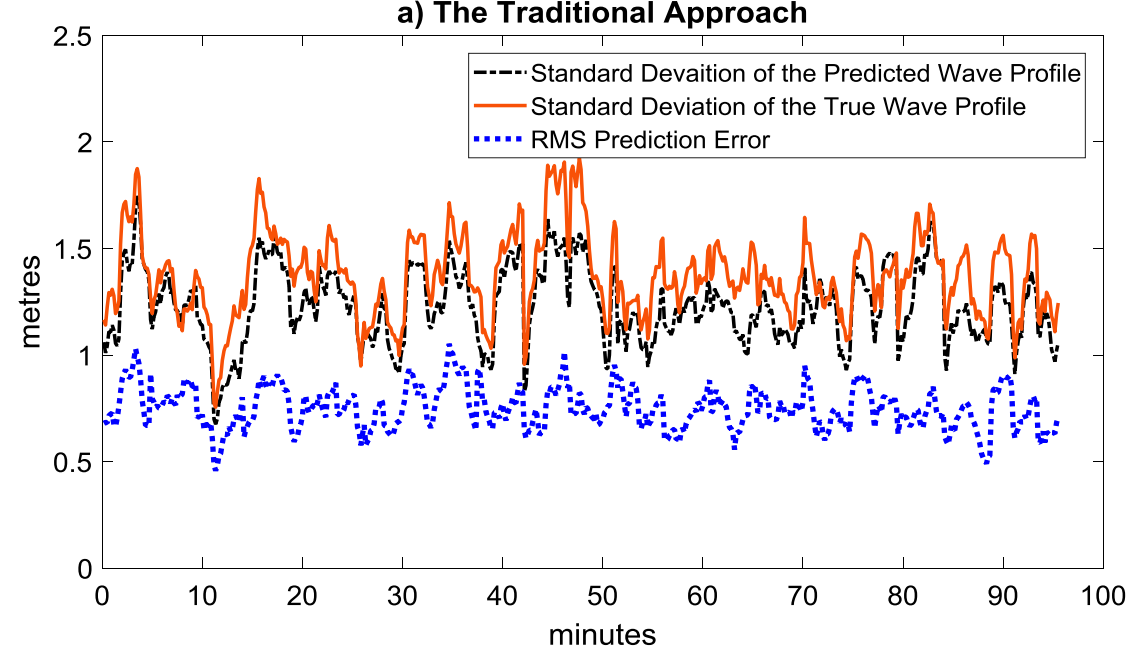

b) The Proposed Technique

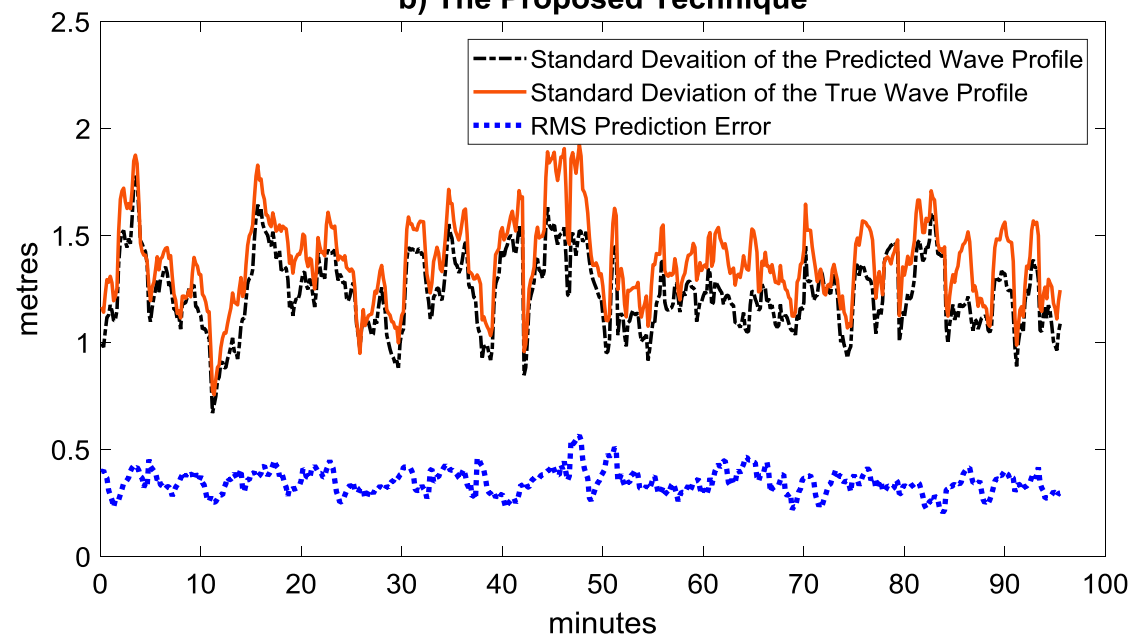

FIG. 2. The RMS prediction error and the standard deviations of the predicted and true wave profiles at the ship location for simulation testing II according to (a) the traditional approach and (b) the proposed technique.

in radial range is introduced in the measurements to further capture the imperfection of the radar. Finally, white Gaussian random noise is added as measurement noise to the generated data resulting in an SNR of $10 \mathrm{~dB}$.

The simulated data cover a 3-km-radius circle with a directional resolution of $0.0063 \mathrm{rad}$ and radial resolution of $3 \mathrm{~m}$. The synthetic scan spans a rotation time $\Delta t$ of $2.5 \mathrm{~s}$. The sea is generated using JONSWAP spectrum as described in the previous subsection. The significant wave height $\mathcal{H}=4.4 \mathrm{~m}$ and the mean period $\mathcal{P}=7.9 \mathrm{~s}$. For the proposed approach, the minimum and maximum wavenumbers are set to 0.001 and $0.16 \mathrm{rad} \mathrm{m}^{-1}$, respectively. The resolutions in wavenumber and angular direction are set to $0.001 \mathrm{radm}^{-1}$ and $0.013 \mathrm{rad}$, respectively. The restricted spectral distribution setups in the proposed approach in the two tests are selected by identifying the maximum dominant wavenumber coefficients and performing the extra self-consistency check, as discussed in section 3c. Nonetheless, this does not represent the optimal size of wavenumbers calculations in both simulation tests. Such optimization would require building a cost function with inputs such as error tolerance, prediction duration and site, and computational complexity. In the case of the traditional methods, the Cartesian grid here is of size $6 \mathrm{~km} \times 6 \mathrm{~km}$ and resolution $1.5 \mathrm{~m} \times 1.5 \mathrm{~m}$. To illustrate the consistency of the prediction quality over the course of time, we run the experiment for $100 \mathrm{~min}$. Using the classical and proposed approaches as described in the previous subsection, predictions of the wave profiles at the ship location for a period of $120 \mathrm{~s}$ are constructed every $10 \mathrm{~s}$. Because of the dead zone around the radar, the 
prediction period based on the scan $/_{s}$ is set to $\left(\tau_{s}+60\right.$, $\left.\tau_{s}+180\right] \mathrm{s}$ to allow the measured waves to propagate to the prediction site. In Figs. 1 and 2, the quality of predictions using the traditional and proposed approaches are shown over $100 \mathrm{~min}$. Figure 1 depicts the correlation coefficients $Q_{s}\left(z_{m}\right)$ between the predicted and the actual wave profiles at the ship location. Figure 2 shows the RMS prediction errors $\varepsilon_{s}\left(z_{m}\right)$ along with the standard deviations of the predicted wave profiles $\hat{\sigma}_{s}\left(z_{m}\right)$, and the standard deviations of the true profiles $\sigma_{s}\left(z_{m}\right)$ at the ship location, for the classical approach in Fig $2 \mathrm{a}$ and the proposed technique in Fig. 2b. The results of the experiment can be summarized as follows:

(i) Under the degraded data, the proposed method using fully time-resolved data still performs significantly better than the traditional two-dimensional Fourier approach using a single time stamp.

(ii) The proposed method is no more vulnerable to the typical measurement errors than the traditional method. And we have no reason to suspect that there are other sources of measurement error that can have particular effect on the proposed method.

(iii) The quality of the prediction according to the proposed method is persistence with time. No significant dropouts are observed in the prediction metrics.

\section{Conclusions}

Ignoring the mixed space-time character of the data in the traditional Fourier-based method constitutes a considerable source of error in DSWP. The proposed technique seems to handle the fully time-resolved wave data very efficiently, eliminating this source of error and considerably outperforming the traditional method. Typical radar measurements artifacts seem to have no particular effect on the proposed technique that cannot be seen on the traditional methods. With the encountered level of error, reasonable computational complexity with no large matrices inversion, and the observed stability, the proposed method constitutes an attractive tool in radar-based DSWP in particular, and spectral applications with rotating scanning technology in general.

Acknowledgments. This work was supported by the EPSRC Research Grant EP/N009142/1.

\section{REFERENCES}

Abusedra, L., and M. Belmont, 2011: Prediction diagrams for deterministic sea wave prediction and the introduction of the data extension prediction method. Int. Shipbuild. Prog., 58, 59-81, https://doi.org/10.3233/ISP-2011-0069.
Alford, L. K., R. F. Beck, J. T. Johnson, D. Lyzenga, O. Nwogu, and A. Zundel, 2015: A real-time system for forecasting extreme waves and vessel motions. Proc. 34th Int. Conf. on Ocean, Offshore and Arctic Engineering, St. John's, NL, Canada, American Society of Mechanical Engineers, 42420, https:// doi.org/10.1115/OMAE2015-42420.

Atanassov, V., W. Rosenthal, and F. Ziemer, 1985: Removal of ambiguity of two-dimensional power spectra obtained by processing ship radar images of ocean waves. J. Geophys. Res., 90, 1061-1067, https://doi.org/10.1029/JC090iC01p01061.

Belmont, M. R., 2010: Increases in the average power output of wave energy converters using quiescent period predictive control. Renewable Energy, 35, 2812-2820, https://doi.org/ 10.1016/j.renene.2010.05.001.

, J. Baker, and J. M. K. Horwood, 2003: Avoidance of phase shift errors in short term deterministic sea wave prediction. J. Mar. Eng. Technol., 2, 21-26, https://doi.org/10.1080/ 20464177.2003.11020172.

, J. M. K. Horwood, R. W. F. Thurley, and J. Baker, 2006: Filters for linear sea-wave prediction. Ocean Eng., 33, 23322351, https://doi.org/10.1016/j.oceaneng.2005.11.011.

,,--- , and -2007 : Shallow angle wave profiling lidar. J. Atmos. Oceanic Technol., 24, 1150-1156, https://doi.org/ 10.1175/JTECH2032.1.

_ J. Christmas, J. Dannenberg, T. Hilmer, J. Duncan, J. M. Duncan, and B. Ferrier, 2014: An examination of the feasibility of linear deterministic sea wave prediction in multidirectional seas using wave profiling radar: Theory, simulation and sea trials. J. Atmos. Oceanic Technol., 31, 1601-1614, https://doi.org/10.1175/JTECH-D-13-00170.1.

Blondel, E., G. Ducrozet, F. Bonnefoy, and P. Ferranti, 2008: Deterministic reconstruction and prediction of non-linear wave systems. Proc. 23rd Int. Workshop Water Waves and Floating Bodies, Jeju, Korea, Seoul National University, 13-16.

Cartwright, D. E., 1963: The use of directional spectra in studying the output of a wave recorder on a moving ship. Proc. Ocean Wave Spectra Conf., Easton, MD, U.S. Naval Oceanographic Office and National Research Council, 203-218.

Connell, B. H., J. P. Rudzinsky, C. S. Brundick, W. M. Milewski, J. G. Kusters, and G. Farquharson, 2015: Development of an environmental and ship motion forecasting system. Proc. 34th Int. Conf. on Offshore Mechanics and Arctic Engineering, St. John's, NL, Canada, American Society of Mechanical Engineers, 42422, https://doi.org/10.1115/OMAE2015-42422.

Crossland, P., J. M. Duncan, and B. Ferrier, 2009: The feasibility of developing a quiescent period prediction system in a simulation environment. Proc. Int. Conf. on Computer Applications in Shipbuilding, Shanghai, China, Royal Institution of Naval Architects, 751-762.

Edgar, D., J. Horwood, R. Thurley, and M. Belmont, 2000: The effects of parameters on the maximum prediction time possible in short term forecasting of the sea surface shape. Int. Shipbuild. Prog., 47, 287-301.

Ferrier, B., A. Baitis, and A. Manning, 2000: Evolution of the landing period designator (LPD) for shipboard air operations. Nav. Eng. J., 112, 297-316, https://doi.org/10.1111/ j.1559-3584.2000.tb03338.x.

_ J. M. Duncan, M. Belmont, A. Curnow, and P. Crossland, 2013: Using simulation to justify and develop quiescent period prediction systems for the Royal Navy. Proc. Int. Conf. on Computer Applications in Shipbuilding, Busan, South Korea, Royal Institution of Naval Architects, 99-107. 
Hilmer, T., and E. Thornhill, 2014: Deterministic wave predictions from the WaMoS II. Proc. OCEANS 2014, Taipei, Taiwan, IEEE, https://doi.org/10.1109/OCEANSTAIPEI.2014.6964526.

$\longrightarrow$, and — 2015: Observations of predictive skill for real-time deterministic sea waves from the WaMoS II. Proc. OCEANS 2015, Washington, DC, IEEE, https://doi.org/ 10.23919/OCEANS.2015.7404496.

Lomb, N. R., 1976: Least-squares frequency analysis of unequally spaced data. Astrophys. Space Sci., 39, 447-462, https://doi.org/ 10.1007/BF00648343.

Longuet-Higgins, M. S., 1984: Statistical properties of wave groups in a random sea state. Philos. Trans. Roy. Soc. London, 312A, 219-250, https://doi.org/10.1098/rsta.1984.0061.

Morris, E., H. Zienkiewicz, M. Pourzanjani, J. Flower, and M. Belmont, 1992: Techniques for sea-state prediction. Proc. Second Int. Conf. on Manoeuvring and Control of Marine Craft, Southampton, United Kingdom, International Federation of Automatic Control, 547-571.

- - - and M. Belmont, 1998: Short-term forecasting of the sea-state. Int. Shipbuild. Prog., 45, 383-400.

Naaijen, P., and A. Wijaya, 2014: Phase resolved wave prediction from synthetic radar images. Proc. 33rd Int. Conf. on Offshore Mechanics and Arctic Engineering, San Francisco, CA, American Society of Mechanical Engineers, 23470, https:// doi.org/10.1115/OMAE2014-23470.

, K. Truslen, and E. Blondel-Couprie, 2014: Limits to the extent of the spatio-temporal domain for deterministic wave prediction. Int. Shipbuild. Prog., 61, 203-233, https://doi.org/ 10.3233/ISP-140113.

Naaijen P., D. K. Roozen, and R. H. M. Huijsmans, 2016: Reducing operational risks by on-board phase resolved prediction of wave induced ship motions. Proc. 35th Int. Conf. on Ocean, Offshore and Arctic Engineering, Busan, South Korea, American Society of Mechanical Engineers, 54591, https://doi.org/10.1115/OMAE2016-54591.
Pourzanjani, M., M. R. Belmont, H. Zienkiewicz, and M. Morris, 1992: Applications of a sea-surface estimator in predictive ship control. Proc. Second Int. Conf. on Manoeuvring and Control of Marine Craft, Southampton, United Kingdom, International Federation of Automatic Control, 581-591.

Prislin, I., J. Zhang, and R. Seymour, 1997: Deterministic decomposition of deep water short-crested irregular gravity waves. J. Geophys. Res., 102, 12 677-12 688, https://doi.org/ 10.1029/97JC00791.

Qi, Y., G. Wu, Y. Liu, and D. K. P. Yue, 2018: Predictable zone for phase-resolved reconstruction and forecast of irregular waves. Wave Motion, 77, 195-213, https://doi.org/10.1016/ j.wavemoti.2017.12.001.

Reichert, K., J. C. Nieto Borge, and J. Dittmer, 1999: Wamos II: A radar based wave and current monitoring system. Proc. Int. Conf. on Ocean and Polar Engineering, Brest, France, International Society of Offshore and Polar Engineers, 139-143.

Scargle, J. D., 1982: Studies in astronomical time series analysis. II. Statistical aspects of spectral analysis of unevenly spaced data. Astrophys. J., 263, 835-853.

Taylor, K. E., 2001: Summarizing multiple aspects of model performance in a single diagram. J. Geophys. Res., 106, 71837192, https://doi.org/10.1029/2000JD900719.

Tucker, M. J., and E. Pitt, 2001: Waves in Ocean Engineering. Ocean Engineering Series, Vol. 5, Elsevier, 548 pp.

—, P. G. Challenor, and D. J. T. Carter, 1984: Numerical simulation of a random sea: A common error and its effect upon wave group statistics. Appl. Ocean Res., 6, 118-122, https:// doi.org/10.1016/0141-1187(84)90050-6.

Vaníček, P., 1969: Approximate spectral analysis by least-squares fit. Astrophys. Space Sci., 4, 387-391, https://doi.org/10.1007/ BF00651344.

Young, I. R., W. Rosenthal, and F. Ziemer, 1985: Three-dimensional analysis of marine radar images for the determination of ocean wave directionality and surface currents. J. Geophys. Res., 90, 1049-1059, https://doi.org/10.1029/JC090iC01p01049. 